\title{
Adaptive Control of Robotic arm with Hysteretic Joint
}

\author{
Somasundar \\ Kannan \\ SnT- University of \\ Luxembourg, \\ L-1359, Luxembourg \\ somasundar.kannan@uni.lu \\ Souad Bezzaoucha \\ SnT- University of \\ Luxembourg, \\ L-1359, Luxembourg \\ souad.bezzaoucha@uni.lu \\ Serket \\ Quintanar-Guzman \\ SnT- University of \\ Luxembourg, \\ L-1359, Luxembourg \\ serket.quintanar@uni.lu \\ Miguel A. \\ Olivares-Mendez \\ SnT- University of \\ Luxembourg, \\ L-1359, Luxembourg \\ miguel.olivaresmendez@uni.lu \\ Holger Voos \\ SnT- University of \\ Luxembourg, \\ L-1359, Luxembourg \\ holger.voos@uni.lu
}

\begin{abstract}
This article addresses the problem of control of robotic arm with a hysteretic joint behaviour. The mechanical design of the one-degree of freedom robotic arm is presented where the joint is actuated by a Shape Memory Alloy (SMA) wire. The SMA wire based actuation of the joint makes the robotic arm lightweight but at the same time introduces hysteresis type nonlinearities. The nonlinear dynamic model of the robotic arm is introduced and an Adaptive control solution is presented to perform the joint reference tracking in the presence of unknown hysteresis behaviour. The Lyapunov stability analysis of the closed loop system is presented and finally proposed adaptive control solution is validated through simulation study on the proposed nonlinear hysteretic robotic arm.
\end{abstract}

\section{CCS Concepts}

- Computer systems organization $\rightarrow$ Robotic control; -Computing methodologies $\rightarrow$ Computational control theory;

\section{Keywords}

Shape Memory Alloy (SMA) Actuator; Robotic Arm; Hysteresis; Adaptive Control.

\section{INTRODUCTION}

Shape Memory Alloy (SMA) actuators are systems using smart materials such as Shape Memory Alloys for the purpose of actuation. The Shape Memory Alloy materials posses certain special properties such as Shape Memory Effect (SME) which is the ability of the material to regain

Permission to make digital or hard copies of all or part of this work for personal or classroom use is granted without fee provided that copies are not made or distributed for profit or commercial advantage and that copies bear this notice and the full citation on the first page. Copyrights for components of this work owned by others than ACM must be honored. Abstracting with credit is permitted. To copy otherwise, or republish, to post on servers or to redistribute to lists, requires prior specific permission and/or a fee. Request permissions from permissions@ acm.org.

ICCMA '16, December 07-11, 2016, Barcelona, Spain

(c) 2016 ACM. ISBN 978-1-4503-5213-0/16/12 . .\$15.00

DOI: http://dx.doi.org/10.1145/3029610.3029632 a predetermined shape on heating. These smart materials have special advantages such as high force to mass ratio, possibility of miniaturization, noiseless operation, and bio-compatibility among others. These properties gives us an alternative solution compared to conventional hydraulic, electric or pneumatic actuators. Different forms of SMA materials can be used for actuation but only the wires-type of SMA actuators will be addressed here in the current article. SMA wires are being used in medical applications [13, 12], dental applications [14], automotive devices [19], SMA based motors [17], robotic manipulators [5, 10, 11] and other general purpose actuators $[3,4,8,7,6,9]$. On the other hand the design and development of SMA actuators are challenging due to low energy efficiency, slow response, hysteresis type nonlinearity which cannot be ignored and requires customized mechanical design and advanced modelling and control solutions.

Different kinds of modelling and control methods have been employed over the years to solve the problem of the hysteresis nonlinearity. The modelling methods can be broadly classified into Phenomenological, Physical, Linear-Model or Model-Free. And depending on the type of models used the control methods can be classified into Inverse Control, Linear-Control or Model-Free-Control and Nonlinear control. Similarly various combination of these control methods can be used with different modelling methods themselves. For extensive survey of modelling and control of SMA actuators please refer to [6].

The focus of the current article is on the control of a Robotic arm actuated by a single SMA wire based joint which was originally designed in [4]. In $[15,16]$ a light weight robotic arm was developed based on the SMA joint model of [4] and sliding mode control was used to perform Joint space control and Operational Space control respectively. Here two SMA wires were used to actuate the joint of the robotic arm.

The key contribution of the current article include development of an Adaptive control approach to control the hysteretic robotic arm in Joint Space. Additionally Lyapunov analysis is performed to study the closed loop properties of the robotic arm with the proposed adaptive control. The current paper is organised as follows: First the mechanical 
model of the robotic arm is briefly described introducing the nonlinear model of the SMA wire along with the kinematics and the dynamics. Next the proposed adaptive control is discussed along with Lyapunov stability analysis. Finally the simulation studies are presented using the proposed adaptive control method.

\section{ROBOTIC ARM WITH SHAPE MEMORY ALLOY (SMA) WIRE BASED JOINT}

In this section we briefly present the design and modelling of the Robotic arm based on Shape Memory Alloy actuated Joint. First the mechanical design of the robotic arm is presented followed by the Dynamic System Modelling. The CAD (Computer Aided Design) model of the robotic arm can be seen in Figure-1. The mechanical design and the system modelling will be further discussed here.

\subsection{Mechanical Design}

The mechanical design of the SMA actuated robotic arm is presented here. The CAD model of the robotic arm can be seen in Figure-1. The key component of the arm design is a rotational joint designed and proposed in [4]. The given joint is an assembly of two couplers named as coupler- 1 and coupler- 2 with a torsional spring in the middle. Further details of the design can be found in [4]. The coupler-1 is attached to an SMA wire and the coupler-2 is attached to a rigid wire. The other ends of both the SMA wire and rigid wire each attached to two separate winding wheels independently which enables the usage of longer wires without increasing the length of the link of the robotic arm. The SMA wire directly affects angular position of the robotic $\operatorname{arm}(\theta)$ through angular displacement of the coupler-1. The couplers in the joints are actuated by SMA wire and rigid wire of equal lengths of $37 \mathrm{~cm}$. The lengths of the links are $100 \mathrm{~mm}$ and $150 \mathrm{~mm}$ which are made of carbon-fibre. The radius of each coupler is $7.5 \mathrm{~mm}$ and the maximum rotational angle is 85 degree in the $x-z$ axis. The total weight of the assembled robotic arm is approximately $45 \mathrm{~g}$.

\subsection{System Modeling}

The complete model of the system can be subdivided into the Actuator model and the Joint controller as seen in Figure-2. The actuator model can be subdivided into model of the SMA wire, Kinematic and Dynamic model of the actuator. The mathematical model of the SMA wire as seen in Figure-3 can be divided into Thermal Dynamics, Phase Transformation and wire Constitutive model. The Geometry and Dynamics of the robotic arm is directly calculated by the simulation environment which is the Simmechanics (Matlab/SIMULINK). The input to the system in closed loop is the angular reference of the Joint $\theta$ given to the controller which generates the required voltage to actuate the SMA based joint. The different components of the system are described in detail now.

\subsubsection{Heat Transfer Model.}

The heat transfer model can be defined by [3, 4]:

$$
m_{w} c_{p} \frac{d T}{d t}=\frac{V^{2}}{R}-h A_{w}\left(T-T_{a m b}\right)
$$

where input voltage is given by $V, R$ is the electric resistance per unit length, the constant specific heat is given by

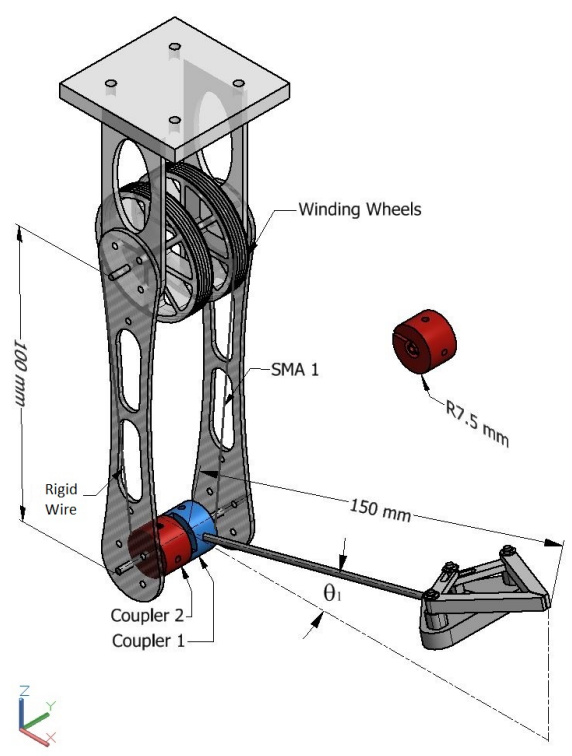

Figure 1: CAD model of the Robotic arm with SMA actuated Joint [15, 16].

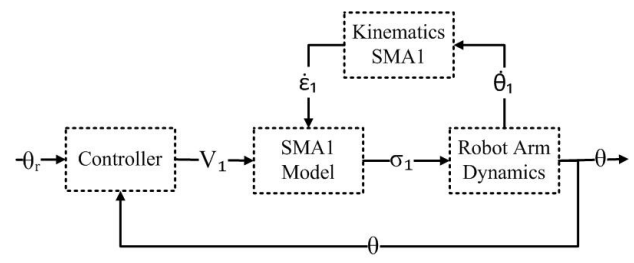

Figure 2: Control scheme of SMA actuated robotic arm

$c_{p}$,mass per unit length is defined by $m_{w}$, the wire surface is defined by $A_{w}, T_{a m b}$ and $T$ the ambient temperature and SMA wire temperature respectively. Here $h$ is the heat convection factor approximated by a second order polynomial of the temperature [4]:

$$
h=h_{0}+h_{2} T^{2}
$$

The heat transfer model defined in (1) uses a constant specific heat $c_{p}$ and is common in literature as seen in $[3,4]$. In the current paper we will use an alternative heat transfer models that also consider the effect of latent heat of transformation $(\Delta H(\sigma))$. The latent heat is modelled as a variation of specific heat of the SMA with respect to temperature. During phase transformation in SMA the heating and cooling are slowed due to the latent heat of transformation [18, 1]. The empirical model of the relationship between specific heat and temperature has been discussed in $[18,1]$. We can now redefine the heat transfer model as follows:

$$
m_{w} c_{p}^{*} \frac{d T}{d t}=\frac{V^{2}}{R}-h A_{w}\left(T-T_{a m b}\right)
$$

where we have for the forward transformation $[18,1]$ :

$$
c_{p}^{*}=c_{p}^{0}+\Delta H(\sigma) \frac{\ln (100)}{\left|M_{s}-M_{f}\right|} \exp \left(-\eta_{1}\right)
$$




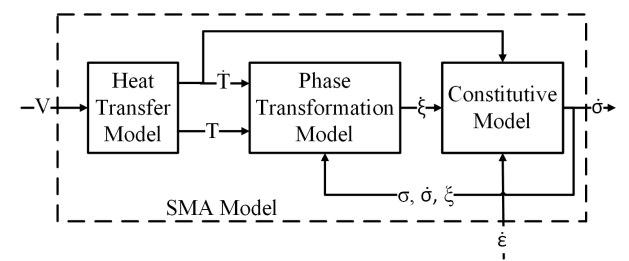

Figure 3: Mathematical Model of the Shape Memory Alloy (SMA) wire $[15,16]$.

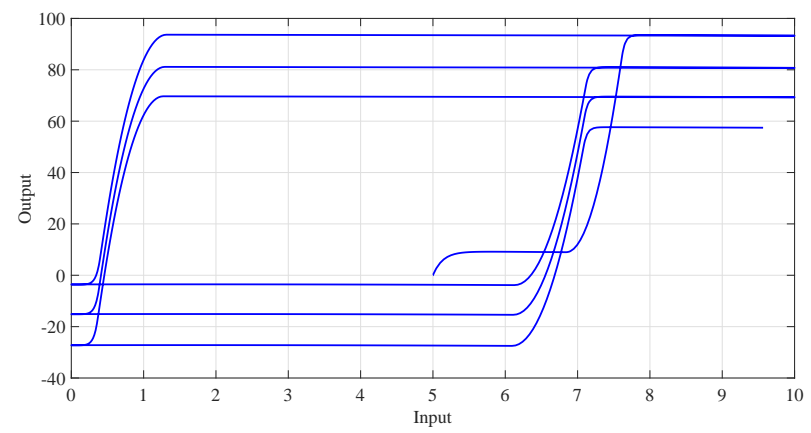

Figure 4: Open-Loop Hysteresis Behaviour in the Robotic Arm

for $M_{f}<T<M_{s}$ and we have $\eta_{1}=\frac{2 \ln (100)}{M_{s}-M_{f}}\left|T-\frac{M_{s}+M_{f}}{2}\right|$. Similarly for reverse transformation we have $[18,1]$ :

$$
c_{p}^{*}=c_{p}^{0}+\Delta H(\sigma) \frac{\ln (100)}{\left|A_{s}-A_{f}\right|} \exp \left(-\eta_{2}\right)
$$

for $A_{f}<T<A_{s}$ and we have $\eta_{2}=\frac{2 \ln (100)}{A_{s}-A_{f}}\left|T-\frac{A_{s}+A_{f}}{2}\right|$. In the above equations we have [18]

$$
\Delta H(\sigma)=\Delta H \times T^{o}(\sigma) / T^{o},
$$

where we have $T^{o}=1 / 2 \times\left(M_{o s}+A_{o s}\right)$ or $T^{o}=1 / 2 \times$ $\left(M_{o f}+A_{o f}\right)$ we calculate $T^{o}(\sigma)=1 / 2 \times\left[M_{s}(\sigma)+A_{s}(\sigma)\right]$. Here $M_{o s}, M_{o f}, A_{o s}, A_{o f}$ are the initial start and finish values for martensite and austenite respectively, $c_{p}^{0}$ is the initial specific heat capacity. In our current model a constant value for $\Delta H=0.06183 \mathrm{~J} \mathrm{~mm}^{-3}$ was used based on [1] where further details of the constant can be obtained.

\subsubsection{SMA Wire Phase Transformation Model.}

Here the phase transformation model is briefly described. The phase transformation model computes the martensite fraction $(\xi)$. The phase transformation model is defined by two set of equations; one for heating and another for cooling. During heating the phase transformation from martensite to austenite is given by [4]

$$
\xi=\frac{\xi_{M}}{2}\left\{\cos \left[a_{A}\left(T-A_{s}\right)+b_{A} \sigma\right]+1\right\}
$$

for temperature $A_{s}+\frac{\sigma}{C_{A}} \leq T \leq A_{f}+\frac{\sigma}{C_{A}}$.

Similarly during cooling the phase transformation from austenite to martensite is described by [4]

$$
\xi=\frac{1-\xi_{A}}{2} \cos \left[a_{M}\left(T-M_{F}\right)+b_{M} \sigma\right]+\frac{1+\xi_{A}}{2}
$$

for temperature $M_{s}+\frac{\sigma}{C_{M}} \leq T \leq M_{f}+\frac{\sigma}{C_{M}}$, where we have $M_{s}, M_{f}, A_{s}, A_{f}$ as the start and end transformation temperatures for martensite and austenite transformation respectively. While we define $a_{A}=\frac{\pi}{\left(A_{f}-A_{s}\right)}, a_{M}=\frac{\pi}{\left(M_{s}-M_{f}\right)}$, $b_{A}=-\frac{a_{A}}{C_{A}}, b_{M}=-\frac{a_{M}}{C_{M}}$, we have $C_{A}$ and $C_{M}$ as the curve fitting parameters.

\subsubsection{Wire Constitutive Model.}

The wire constitutive model is given by [3]

$$
\dot{\sigma}=E \dot{\varepsilon}+\Omega \dot{\xi}+\Theta \dot{T}
$$

where, $\sigma$ is the stress, $\varepsilon$ is the strain, $T$ is the temperature and $\xi$ is the martensite fraction. Here $\Omega$ and $\Theta$ are the phase transformation constant and thermal expansion coefficient respectively, while $\Omega=-E \varepsilon_{0}$ with $\varepsilon_{0}$ given as initial strain. The young's modulus is given by

$$
E=\xi E_{M}+(1-\xi) E_{A}
$$

where $E_{A}$ is the average young's modulus of austenite phase and $E_{M}$ is the average young's modulus of martensite phase.

\subsubsection{Kinematics and Dynamics.}

In this section the model of the mechanical design and its relation with the rest of the system is explained.

Kinematic Model. The kinematics of the SMA actuator is described through the strain rate of the SMA wire

$$
\dot{\varepsilon}=-\frac{r \dot{\theta}}{l_{0}}
$$

where $r$ is the radius of the coupler, $l_{0}$ the initial length of the SMA wire and $\dot{\theta}$ the angular velocity of the coupler. Here it should be noted that only the coupler- 1 is rotating and the coupler- 2 is attached to a rigid wire.

Dynamics. The general dynamic model of the SMA actuated robotic arm can be given by

$$
A(\theta) \ddot{\theta}+B(\theta, \dot{\theta}) \dot{\theta}+g(\theta)+C \dot{\theta}+\Phi\left(\theta, \theta_{r}\right)=\tau_{w}
$$

where $\theta, \dot{\theta}, \ddot{\theta}$ represents the position, velocity and acceleration of the systems, $A(\theta)$ is the inertia matrix, $B(\theta, \dot{\theta})$ is the centripetal-coriolis matrix, $g(\theta)$ is considered as the effect of gravity, $C$ is the viscous coefficient term, $\Phi\left(\theta, \theta_{r}\right)$ is the nonlinear hysteretic term, $\tau_{w}$ is the input torque applied to the manipulator joint by the SMA wire given by

$$
\tau_{w}=F_{w} r=A_{c} \sigma r
$$

where $\sigma$ is the stress in the SMA wire, $r$ is the radius of the coupler, $A_{c}$ is the transversal area of the coupler. The effect of torsional spring is parametrized and included in $B(\theta, \dot{\theta})$ by the function $k_{s} \theta+k_{d} \dot{\theta}$ where $k_{s}$ is the spring constant and $b_{s}$ is the spring friction constant. The nonlinear hysteresis behaviour present in the robotic arm can be seen in Figure-4. The input output behaviour seen in the Figure- 4 is relation between input reference $\theta_{r}$ and the output response $\theta$ of the arm Joint.

The complete dynamics of the robotic arm as described before can be composed of rigid and moving parts designed and assembled using CAD (see Figure-1). This CAD model is then imported into Simmechanics environment of Matlab/SIMULINK. The Nonlinear Model of the SMA is computed mathematically and integrated into the Simmechanics 
environment. In the following sections we will introduce the control method used and finally perform simulation studies on the given dynamic model of the robotic arm.

\section{ADAPTIVE CONTROL}

In the section we will briefly present the adaptive control solution based on [2]. Let $e(t) \in \mathbb{R}^{n}$ be the joint tracking error

$$
e=\theta_{d}-\theta
$$

where $\theta_{d}(t) \in \mathbb{R}^{n}$ is the desired joint angle. We further introduce the filtered tracking error $r(t) \in \mathbb{R}^{n}$ given as [2]

$$
r=\dot{e}+\alpha e
$$

where $\alpha$ is a constant gain. Now similar to the computation in [2] after taking derivative of (15) with respect to time and multiplying with $A(\theta)$ on both sides we get

$$
A(\theta) \dot{r}=A(\theta)\left(\ddot{\theta}_{d}+\alpha \dot{e}\right)-A(\theta) \ddot{\theta} .
$$

Now making substitution for $A(\theta) \ddot{\theta}$ we get

$$
\begin{aligned}
A(\theta) \dot{r}= & A(\theta)\left(\ddot{\theta}_{d}+\alpha \dot{e}\right)+B(\theta, \dot{\theta})\left(\dot{\theta}_{d}+\alpha e\right)+g(\theta)+C \dot{\theta} \\
& -B(\theta, \dot{\theta}) r+\Phi\left(\theta, \theta_{r}\right)-\tau
\end{aligned}
$$

After algebraic manipulation [2] the open loop dynamics in terms of $r$ can be written as

$$
A(\theta) \dot{r}=-B(\theta, \dot{\theta}) r+\beta-\tau
$$

where we have

$\beta=A(\theta)\left(\ddot{\theta}_{d}+\alpha \dot{e}\right)+B(\theta, \dot{\theta})\left(\dot{\theta}_{d}+\alpha e\right)+g(\theta)+C \dot{\theta}+\Phi\left(\theta, \theta_{r}\right)$

Based on the above open loop dynamics let us choose the control input

$$
\tau=\hat{\beta}+K r
$$

where $K$ is the control gain and $\hat{\beta}$ is the estimate of unknown $\beta$. The value of $\hat{\beta}$ is updated using the update law

$$
\dot{\hat{\beta}}=\Gamma^{-1} r
$$

where $\Gamma$ is a positive definite adaptation gain. Using (18) and (20) we can define the closed loop system in terms of $r$ as follows

$$
A(\theta) \dot{r}=-B(\theta, \dot{\theta}) r-K r+\tilde{\beta}
$$

where $\tilde{\beta}=\beta-\hat{\beta}$. In order to study the stability properties let us first choose the Lyapunov function [2]

$$
V=\frac{1}{2} r^{T} A(\theta) r+\frac{1}{2} \tilde{\beta}^{T} \Gamma \tilde{\beta} .
$$

Differentiating $V$ with respect to time we get

$$
\dot{V}=\frac{1}{2} r^{T} \dot{A}(\theta) r+r^{T} A(\theta) \dot{r}+\frac{1}{2} \tilde{\beta}^{T} \Gamma \dot{\tilde{\beta}} .
$$

Now we substitute the closed loop equation (22) in to the above equation to get

$$
\dot{V}=\left[\frac{1}{2} r^{T} \dot{A}(\theta) r-r^{T} B(\theta, \dot{\theta}) r\right]-r^{T} K r+\tilde{\beta}^{T}(r+\Gamma \dot{\tilde{\beta}}) .
$$

Let us use the property $\frac{1}{2} r^{T} \dot{A}(\theta) r-r^{T} B(\theta, \dot{\theta}) r=0$ [2] and then substitute $\dot{\tilde{\beta}}=-\Gamma^{-1} r$ (since $\beta$ is a constant by assumption). Then by further simplification we get

$$
\dot{V}=-r^{T} K r \leq-\lambda_{\min }\{K\}\|r\|^{2}<0
$$

Table 1: Simulation Parameters $[4,3]$

\begin{tabular}{cllc}
\hline Parameter & Value & Parameter & Value \\
\hline$E_{M}$ & $28 \mathrm{GPa}$ & $C_{A}$ & $10 \mathrm{Mpa} /{ }^{\circ} \mathrm{K}$ \\
$E_{A}$ & $75 \mathrm{GPa}$ & $C_{M}$ & $10 \mathrm{Mpa} /{ }^{\circ} \mathrm{K}$ \\
$A_{s}$ & $88^{\circ} \mathrm{C}$ & $T_{a m b}$ & $25^{\circ} \mathrm{C}$ \\
$A_{f}$ & $98^{\circ} \mathrm{C}$ & $A$ & $4.9 x 10^{-8} \mathrm{~m}^{2}$ \\
$M_{s}$ & $72^{\circ} \mathrm{C}$ & $A_{w}$ & $290.45 x 10^{-6} \mathrm{~m}^{2}$ \\
$M_{f}$ & $62^{\circ} \mathrm{C}$ & $c_{p}$ & $320 \mathrm{~J} / \mathrm{Kg}^{\circ} \mathrm{C}$ \\
$m_{w}$ & $6.8 x 10^{-4} \mathrm{~kg} / \mathrm{m}$ & $\varepsilon_{L}$ & $2.3 \%$ \\
$R$ & $20 \Omega / m$ & $h_{0}$ & 20 \\
$l_{0}$ & $0.37 \mathrm{~m}$ & $h_{2}$ & 0.001 \\
$b_{s}$ & 0.5 & $b_{1}, b_{2}$ & 0.1 \\
$k_{s}$ & $0.0018 \mathrm{Nm} / 1^{o}$ & $\Theta$ & -0.055 \\
\hline
\end{tabular}

where $\lambda_{\min }\{\cdot\}$ is the minimum eigen value of the matrix. From the above equation (26) we can conclude that the closed loop system using control law (20) and adaptation law (21) is asymptotically stable since the time derivative of the Lyapunov function is negative.

\section{CONTROL SIMULATION OF SMA BASED ROBOTIC ARM}

Here we will briefly address the simulation study of the above discussed adaptive control law by application to joint space control of the hysteretic manipulator. The nonlinear model of the robotic arm with SMA joint is used along with parameter set in Table-1. During this simulation the control law in (20) is used along with the adaptation law (21). The controller parameters used in the simulation are $K=10$, $\alpha=2$ and $\Gamma=0.2$. The simulation results can be seen in Figure-5, Figure-6.

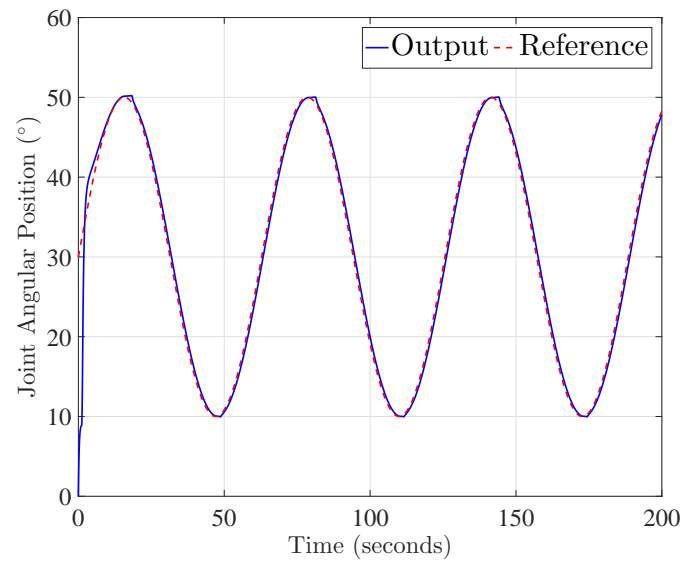

Figure 5: Joint Angular Position Tracking in the Robotic Arm

The closed loop position control response of the hysteretic joint can be seen in Figure-5. The control input can be seen in Figure-6. In Figure-5 we can see that the adaptive control can compensate for the unknown hysteresis nonlinearity and achieve satisfactory reference tracking of a sinusoidal signal. In Figure-6 we can see that the control signal is restricted to $0-10$ volts. This is done in order to avoid excessive heating of the SMA wire, which is common in experimental conditions. Although the input constraint is added for the 


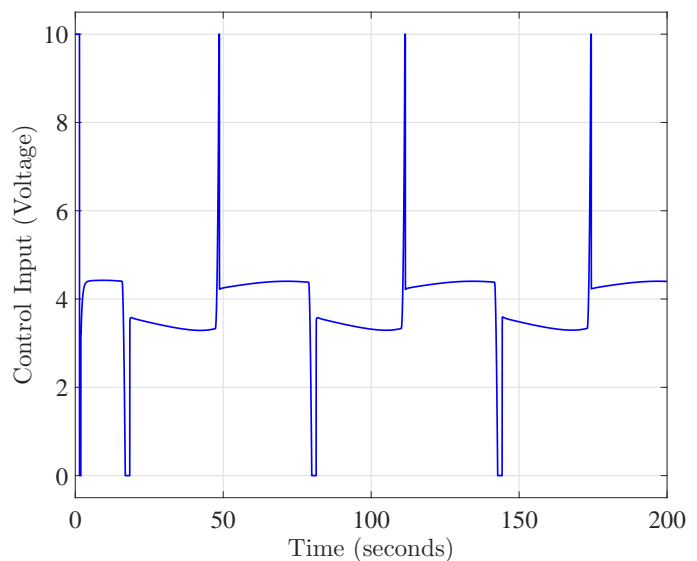

Figure 6: Joint Angular Position Tracking: Control Input

safety of the SMA wire, this also introduces an additional saturation nonlinearity.

\section{CONCLUSION}

In the current paper we briefly discussed the design and modelling of a single degree of freedom robotic arm actuated by SMA wire. The hysteresis nonlinearity in the robotic arm was addressed first and then an Adaptive control solution was proposed with Lyapunov stability analysis. The simulation studies successfully presented the application of an adaptive control solution to control a hysteretic robotic arm. The future perspectives of the current work is to construct the proposed robotic arm and validate the discussed adaptive control solution.

\section{REFERENCES}

[1] A. Bhattacharyya, D. C. Lagoudas, Y. Wang, and V. K. Kinra. On the role of thermoelectric heat transfer in the design of sma actuators: theoretical modeling and experiment. Smart Materials and Structures, 4(4):252, 1995.

[2] M. S. de Queiroz, D. M. Dawson, S. P. Nagarkatti, and F. Zhang. Lyapunov-Based Control of Mechanical Systems. Springer Science \& Business Media, 2012.

[3] M. H. Elahinia and H. Ashrafiuon. Nonlinear Control of a Shape Memory Alloy Actuated Manipulator. Journal of Vibration and Acoustics, 124(4):566, 2002.

[4] Z. Guo, Y. Pan, L. B. Wee, and H. Yu. Design and control of a novel compliant differential shape memory alloy actuator. Sensors and Actuators A: Physical, 225:71-80, apr 2015.

[5] M. Hulea and C. F. Caruntu. Spiking neural network for controlling the artificial muscles of a humanoid robotic arm. pages 163-168, 2014.

[6] S. Kannan. Modélisation et Commande d'Actionneurs à Alliage à Mémoire de Forme. $\mathrm{PhD}$ thesis, l'École Nationale Supérieure d'Arts et Métiers, 2011.

[7] S. Kannan, C. Giraud-Audine, and E. Patoor. Control of shape memory alloy (sma) actuator using series-parallel model reference adaptive control (mrac). In ASME 2009 Conference on Smart
Materials, Adaptive Structures and Intelligent Systems, pages 441-450. American Society of Mechanical Engineers, 2009.

[8] S. Kannan, C. Giraud-Audine, and E. Patoor. Laguerre model based adaptive control of antagonistic shape memory alloy (sma) actuator. In SPIE Smart Structures and Materials + Nondestructive Evaluation and Health Monitoring, pages 764307-764307. International Society for Optics and Photonics, 2010.

[9] S. Kannan, C. Giraud-Audine, and E. Patoor. Application of laguerre based adaptive predictive control to shape memory alloy (sma) actuator. ISA Transactions, 52(4):469 - 479, 2013.

[10] A. Khodayari, M. Talari, and M. M. Kheirikhah. Fuzzy PID controller design for artificial finger based SMA actuators. IEEE International Conference on Fuzzy Systems, pages 727-732, 2011.

[11] J. Ko, M. B. Jun, G. Gilardi, E. Haslam, and E. J. Park. Fuzzy PWM-PID control of cocontracting antagonistic shape memory alloy muscle pairs in an artificial finger. Mechatronics, 21(7):1190-1202, 2011.

[12] X. Liu, Y. Wang, D. Yang, and M. Qi. The effect of ageing treatment on shape-setting and superelasticity of a nitinol stent. Materials Characterization, 59(4):402-406, 2008.

[13] F. Nematzadeh and S. Sadrnezhaad. Effects of material properties on mechanical performance of nitinol stent designed for femoral artery: Finite element analysis. Scientia Iranica, 19(6):1564 - 1571, 2012.

[14] N. Pandis and C. P. Bourauel. Nickel-titanium (niti) arch wires: the clinical significance of super elasticity. In Seminars in Orthodontics, volume 16, pages 249-257. Elsevier, 2010.

[15] S. Quintanar-Guzman, S. Kannan, M. A. Olivares-Mendez, and H. Voos. Lightweight robotic arm actuated by shape memory alloy (sma) wires. In IEEE International Conference on Electronics, Computers and Artificial Intelligence, pages -. IEEE, 2016.

[16] S. Quintanar-Guzman, S. Kannan, M. A. Olivares-Mendez, and H. Voos. Operational space control of a light weight robotic arm actuated by shape memory alloy (sma) wires. In Smart Materials, Adaptive Structures and Intelligent Systems (ASME-SMASIS 2016), pages -. ASME, 2016.

[17] S. Quintanar-Guzmán, J. Reyes-Reyes, and M. d. c. Arellano-Sánchez. Modelado y control de un sistema electrotérmico-mecánico móvil basado en alambres musculares. In XVI Congreso Latinoamericano de Control Automático, CLCA 2014, pages 834-839. Asociación de México de Control Automático, 2014.

[18] O. K. Rediniotis, D. C. Lagoudas, H. Y. Jun, and R. D. Allen. Fuel-powered compact sma actuator, 2002.

[19] E. A. Williams, G. Shaw, and M. Elahinia. Control of an automotive shape memory alloy mirror actuator. Mechatronics, 20(5):527-534, 2010. 